\title{
BLOWFISH ALGORITHM-SECURING THE FUTURE
}

\author{
U.V. Ratna Kumari ${ }^{1 *}$, Santosh Pokhrel ${ }^{2}$, Hyndavi Anusha Anche ${ }^{3}$ \\ *1,2,3 Assistant Professor in JNTUK, Student-B.Tech, Student-B.Tech ECE DEPT.,UCEK, JNTUK, KAKINADA \\ *1vinayratna74@gmail.com, ${ }^{2}$ santosh_everest@yahoo.com, ${ }^{3}$ hyndavianche@gmail.com
}

*Corresponding Author: -

Email: vinayratna74@gmail.com

\begin{abstract}
:
Information security has become a major issue in communication systems. Any threat to the information being transferred may cause severe loss to the organization. To overcome this security threat cryptography techniques are used to encrypt and decrypt the data. Many algorithms are used for this purpose, one among them is the blowfish algorithm. This paper presents a detail regarding the encryption and decryption using blowfish algorithm using VHDL coding and its post simulation results. The beauty of this algorithm lies in its prepossessing way of generating the sub-keys.
\end{abstract}

Keywords: Encryption, Decryption, Plain text, Cipher text, Blowfish, P-array, Sub keys, S-box

\section{(c) (\$) (1)}




\section{I.INTRODUCTION}

The main purpose of cryptographic algorithms is to provide security services such as authentication, access control, data confidentiality, data integrity and non-repudiation.

Authentication: The assurance that the communicating entity is the one that it claims to be.

Access control: The prevention of unauthorized use of resource.

Data confidentiality: The protection of data from unauthorized disclosure.

Data integrity: The assurance that data received is exactly as sent by an authorized entity.

Non repudiation: Prevents either sender or receiver from denying a transmitted message.

Various terms used in cryptography are: Plain text: This is the original intelligible message or data that is fed into the algorithm as input. Secret Key: It is also an input to the algorithm whose output varies with the key.

Cipher text: The modified message obtained at the output depending on the plain text and the secret key.

Encryption algorithm: This performs various substitutions and transformations on the plain text to

obtain the cipher text.

Decryption algorithm: This is essentially the encryption algorithm run in reverse. It uses the Cipher text and secret key to obtain the plain text.

\section{CLASISIFICATION OF CRYPTOGRAPHIC ALGORITHMS}

Cryptographic algorithms can be broadly classified into two types: Symmetric algorithms and Asymmetric algorithms. Symmetric algorithms have a single private key which is known to the sender and the receiver whereas asymmetric algorithms have two keys, a public key and a private key. Symmetric algorithms can be further divided into block ciphers and stream ciphers. Block ciphers operates on fixed lengths of groups of input data whereas stream ciphers encode streams of data. DES, IDEA, RC5, AES, Blowfish are some types of block ciphers. RC4, ISAAC, Grain are some types of stream ciphers. DSS, RSA encryption algorithms come under asymmetric algorithms.

\section{III.BLOWFISH ALGORITHM DESCRIPTION}

Blowfish is a symmetric key crypto system which is based on a Feistel network. The algorithm was developed by Bruce Schneier in 1993. Blowfish is a block cipher with a block size of 64 bits, and the full version uses 16 rounds to complete the encryption of a block. Blowfish is unpatented and license-free, and is available free for all uses. The block size is 64 bits, and the secret key can vary from 32 bits to 448 bits. This algorithm used large number of sub-keys during encryption and decryption. The P-array consists of 1832 bit sub keys: P1,P2,..,P18.

There are four 32-bit S-boxes with 256 entries each:

$\mathrm{S} 1,0, \mathrm{~S} 1,1, \ldots, \mathrm{S} 1,255$

$\mathrm{S} 2,0, \mathrm{~S} 2,1, \ldots, \mathrm{S} 2,255$

$\mathrm{S} 3,0, \mathrm{~S} 3,1, \ldots, \mathrm{S} 3,255$

$\mathrm{S} 4,0, \mathrm{~S} 4,1, \ldots, \mathrm{S} 4,255$.

Figure1: Encryption routine for blowfish are utilized. The values are stored in ROM are extracted from hexadecimal representation of pi(post decimal digits). First 32 bits are assigned to P1, next to P2 and so on.

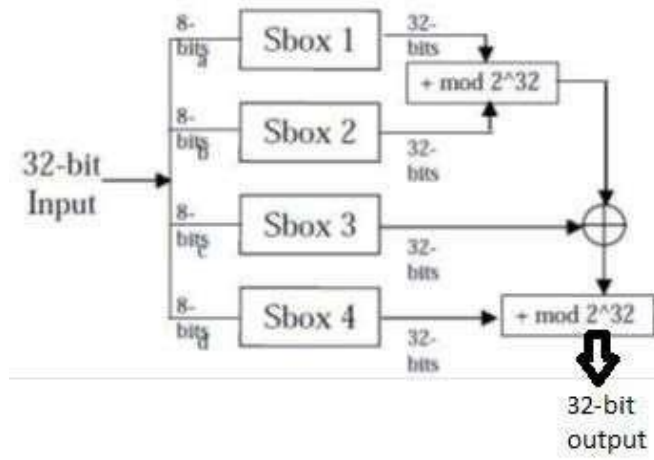

Similarly, S-box used the ROM size of $\left(2^{\wedge} 8^{*} 32\right), 8$ address lines and 256 data lines each of 32 bits. The value assignment follows the same manner like in P-array. In summary, $\mathrm{S}$ box maps the address input to corresponding output. The input is text dependent. The design uses a structural style of modeling to initialize and perform its encryption/decryption functionality. 
The graphical representation of blowfish algorithm is shown in the following figures.

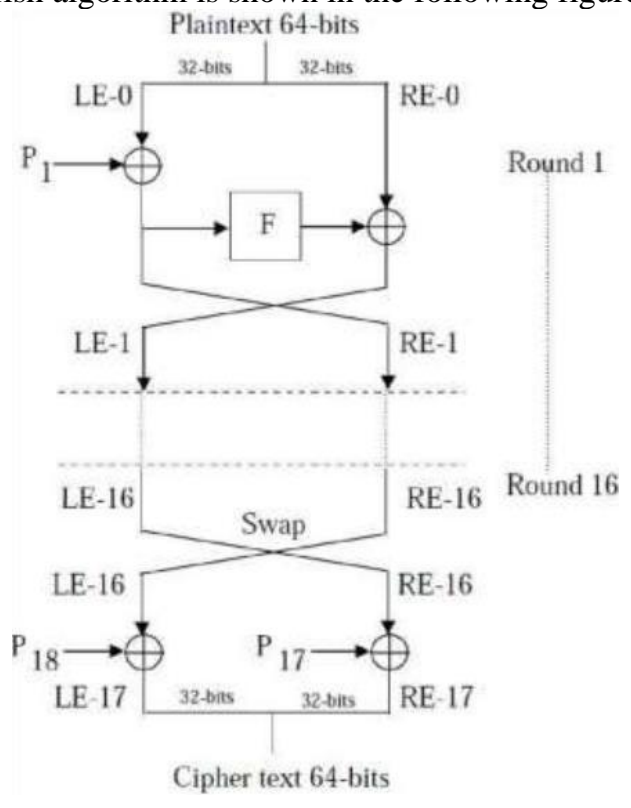

1. Initialize first the P-array and then the four Sboxes, in order, with a fixed string. This string consists of the hexadecimal digits of pi (less the initial 3): P1 =0x243f6a88, P2 = 0x85a308d3, P3 = Figure 2: Internal operation of $F$

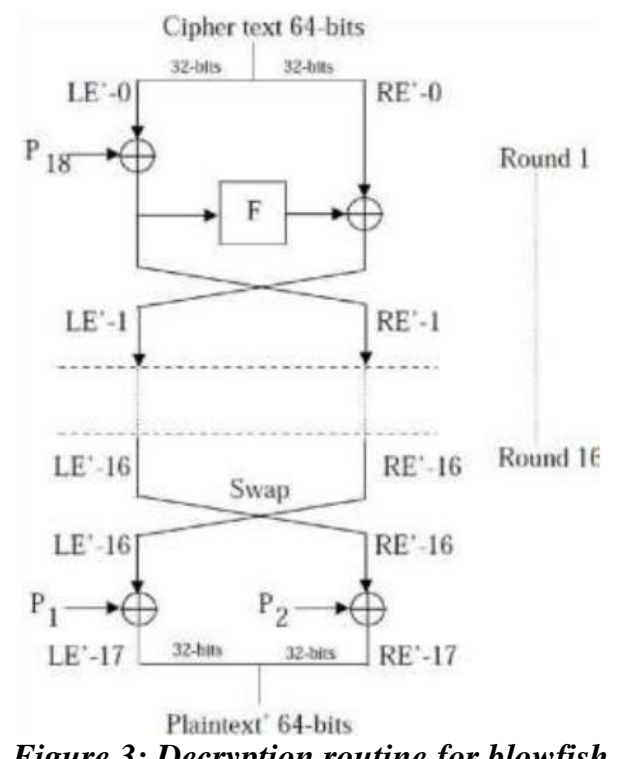

function 0x13198a2e, P4 = 0x03707344, etc.

2. XOR P1 with the first 32 bits of the secret key,

3. Then modified P1 is xored with left half of plain text.

4. The result obtained from process 3 is fed to fiestel network. The $8 * 4$ data acts as address for $\mathrm{S}$ boxes, which then generate 32 bits corresponding output.

5. The output of S1 and S2 undergo 32 bits moduloaddition, the output then xored with the S3 output and finally the result performs 32 bit modulo addition with $\mathrm{S} 4$ output.

6. This 32 bit output obtained from process 4 is then xored with right half of plain text.

7. The two results obtained from process (3) and process (5) are then swapped.

8. This continues for 15 more iterations.

9. Then the final cipher text is generated by completing the remaining process as shown in encryption routine fig(1).

\section{Proposed Design:}

Our design consists of several ROMs to store the set of values of P-array and S-boxes. P-array uses the ROM size of $\left(2^{\wedge} 5^{*} 32\right), 5$ address lines and 32 data lines each of 32 bits; out of which only 18 data lines

XOR P2 with the second 32-bits of the key, and so on for all bits of the key (possibly up to P14). Repeatedly cycle through the key bits until the entire P-array has been XORed with key bits. 


\section{Glimpses of Simulation:}

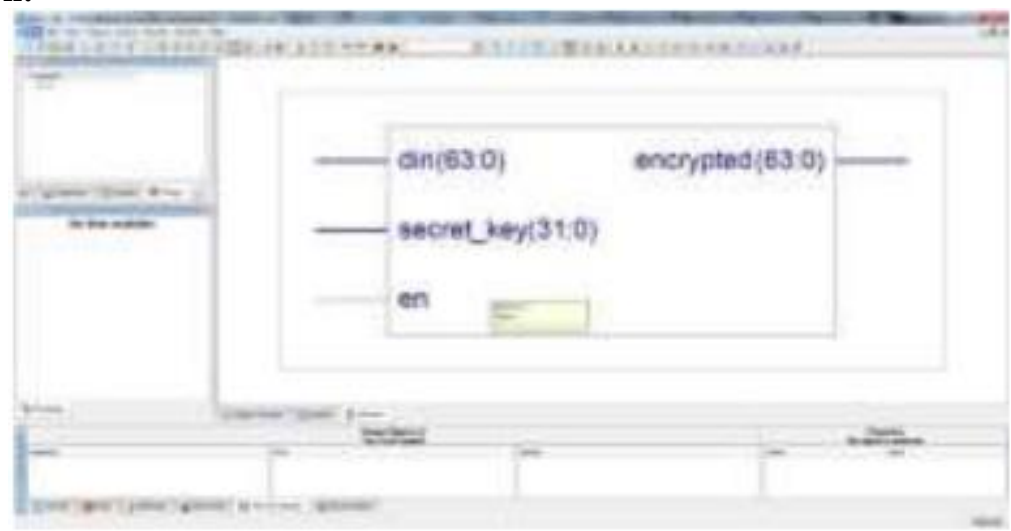

Figure 4: RTL Synthesis for 32 bits secret key

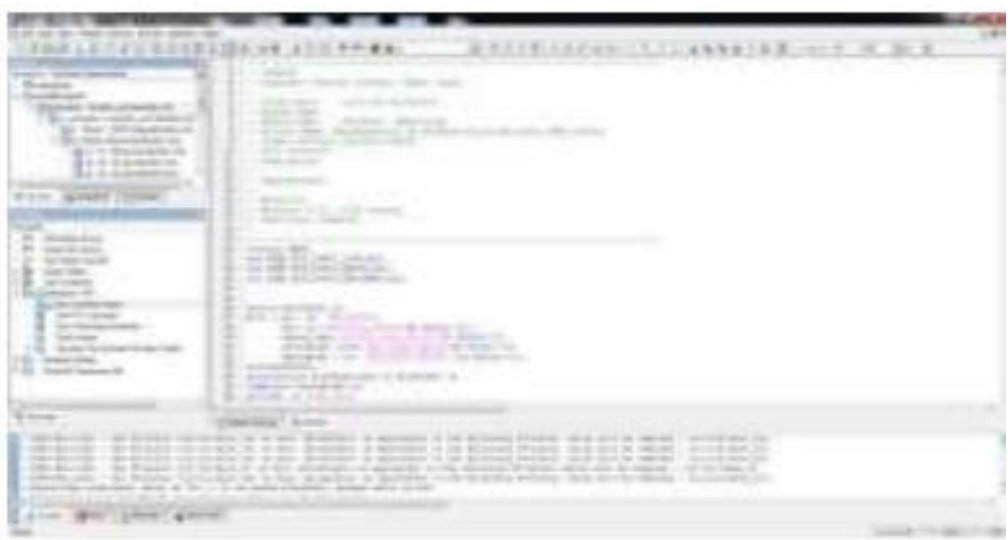

Figure 5: Screen shot of synthesis in Xilinx 8.1

\begin{tabular}{|l|l|l|l|l|}
\hline $\begin{array}{l}\text { Algorith } \\
\text { m }\end{array}$ & Type & $\begin{array}{l}\text { Clocks/ } \\
\text { Round }\end{array}$ & \#Round & $\begin{array}{l}\text { Clock/By } \\
\text { te of } \\
\text { output }\end{array}$ \\
\hline RC4 & $\begin{array}{l}\text { Stream } \\
\text { cipher }\end{array}$ & n. a & n. a & 7 \\
\hline SEAL & $\begin{array}{l}\text { Stream } \\
\text { cipher }\end{array}$ & n. a. & n. a & 4 \\
\hline Blowfish & $\begin{array}{c}\text { Block } \\
\text { Cipher }\end{array}$ & 9 & 16 & 18 \\
\hline RCS & $\begin{array}{l}\text { Block } \\
\text { Cipher }\end{array}$ & 12 & 16 & 23 \\
\hline DES & $\begin{array}{l}\text { Block } \\
\text { Cipher }\end{array}$ & 18 & 16 & 45 \\
\hline IDEA & $\begin{array}{l}\text { Block } \\
\text { Cipher }\end{array}$ & 50 & 8 & 50 \\
\hline Triples & $\begin{array}{l}\text { Block } \\
\text { Cipher }\end{array}$ & 18 & 48 & 108 \\
\hline
\end{tabular}

Table 1: Block and Stream Cipher Speed Comparison

\section{Result:}

Our proposed design has been implemented and it could generate the correct cipher text for all 64 bits plain text with variable length of secret key. The plain text has been successfully recovered using the decryption routine shown in fig 2 . The synthesis report has been generated for the target device $\mathrm{xc} 3 \mathrm{~s} 500 \mathrm{e}-4 \mathrm{fg} 320$ with the utilization of $37 \%$ available slices (1731/4656 slices). 


\section{IV.CONCLUSION}

This is the simple and easy method of implementation of Blowfish algorithm utilizing minimum computing resources. Most of the computer users and even layman can make best use of this algorithm for securing the future.

\section{REFERENCES}

[1].B. Schneier, Description of a New Variable- Length Key, 64-Bit Block Cipher (Blow- fish), Fast Software Encryption, Cambridge Security Workshop Proceedings (December 1993), Springer-Verlag, 1994.

[2].Afaf M. Ali Al-Neaimi, New Approach for Modifying Blowfish Algorithm by Using Multiple Keys, IJCSNS, March 2011.

[3].B. Schneier, “Applied Cryptography,” 2nd ed. New York: , JohnWiley \& Sons, Inc., 1996.

[4].B. Schneier, "Description of a New Variable Length Key, 64-bit Block Cipher (Blowfish)," Fast Software Encryption: Second International Workshop, Leuven, Belgium, December 1994, Proceedings, SpringerVerlag, 1994, pp. 191-204.

[5].Mr. Tushar Joshi "Design of enhanced speed blowfish algorithm for cryptography with merged encryption \& decryption in VHDL" 